\title{
A Case Study for Choosing Proper Relocation Algorithms to Recover Large Scale Coverage Hole(s) in Wireless Sensor Networks
}

\author{
Ali Rafiei, Mehran Abolhasan, Daniel Franklin \\ Centre for Real-time Information Networks (CRIN), \\ Faculty of Engineering and Information Technology \\ University of Technology, Sydney,Sydney, Australia \\ Email: \{ali.rafiei,mehran.abolhasan,daniel.franklin\}@uts.edu.au \\ Stephen Smith \\ Faculty of Science, Department of Computing, Macquarie University, Sydney, Australia \\ Email: stephen.smith@mq.edu.au
}

\begin{abstract}
Coverage holes as large scale en mass and correlated node failures in wireless sensor networks, not only disturb the normal operation and functionality of networks, but also may endanger network's integrity. Recent trends to use relocation of currently deployed nodes have attracted attention especially where manual addition of nodes are neither feasible nor economical in many applications. The transition from centralized to distributed node relocation algorithm gradually paves away for applications in which nodes are deployed in harsh and hostile environments in absence of central supervision and control. Although, many different relocation algorithms have been devised to address their given applications' challenges and requirements and they are efficient in reaching their design goals, they may not be similarly responsive to unpredicted and different circumstances may occur in the network. This paper, demonstrates one of such case, DSSA (Distributed Self-Spreading Algorithm) that is mainly applied for balancing node deployments and recovery of small coverage holes. It is shown here that DSSA is not able to fully recover large scale coverage holes even if all nodes participate in recovery process and relocate with sufficient number of iterations.
\end{abstract}

\section{INTRODUCTION}

With constant advancements in sensor nodes, wireless sensor networks (WSNs) [1], [2] have been adapted for many applications [3]-[9] especially in harsh and hostile environment where there is lack of centralized supervision and control. With increasing flexibility and ubiquity (i.e. in structures [10], underground [11], in the air [12], underwater [13], or on the surface of sea [14]) come new challenges and design requirements which should be properly addressed in wireless sensor networks in their designated applications. Coverage holes $(\mathrm{CHs})$ as en masse and correlated failures of deployed sensor nodes [15] as the result of battery exhaustion or physical damages [16] is one of prevalent issues which should be dealt with accordingly, otherwise they not only disturb the normal operation of network but also jeopardize the integrity of whole network if they remain unattended at their occurrences.

Benefiting from controlled mobility of nodes [17], [18], relocation of currently deployed nodes are used to recover the coverage holes and unbalanced network formations seems to be promising solution especially where neither centralized control nor manually addition of nodes deem feasible. Thus, to properly exploit available redundancy of deployed nodes, many relocation algorithms have been introduced in order to repair and make network more flexible and robust in failureprone environments with the harsh and hostile conditions.

In spite of recent advancements in batteries and energyScavenging technologies and distributed relocation algorithms, physical movements especially in autonomous nodes, consume majority of nodes' energy. Inspired by the nature [19], many relocation algorithms devised [18] to address the emerging challenges caused by transition from centralized to distributed control of network and to make objective movements of node movements more practical and implementable in large scale networks. Although the distributed relocation algorithms mainly evolved by reducing the consumed energy of nodes, they may not be similarly efficient and responsive to unpredictable events and circumstances which may occur in the network and are not accounted for in the relocation algorithms. In this paper, it is shown that although DSSA relocation algorithm is rather efficient to repair and recover small coverage holes and to balance network formations, it is not a proper choice as it is not able to properly recover large scale coverage holes with reasonable number of iterations even if all nodes participate in recovery process.

The paper is organized as follows: in Section II related work are presented. In Section III, the methods and assumptions are introduced. In Section IV, the results are demonstrated, and finally the paper is concluded in Sections V conclusion.

\section{RELATED WORK}

In order improve the coverage and nodes formation, among the solutions, relocation of currently deployed nodes offers a promising solution to emerging issues and challenges in wireless sensor networks. By harnessing the controllable and constrained node mobility a wide variety of relocation algorithms have been introduced in the literature [20]-[32] to 


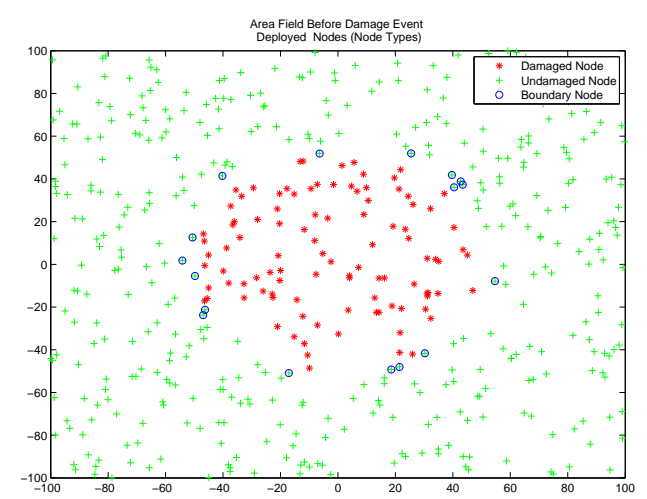

Fig. 1: Network Hole and Node Types

deal with unbalanced deployment and newly formed coverage holes, and to dynamically response to unpredictable topological changes in wireless sensor networks. By the advantage of WSNs' intrinsic redundancy, distributed relocation algorithms seems to be proper candidates for applications with lack of centralized control and supervision with harsh environments. In such environments, autonomous nodes should be able use their neighbours' status in order to make decision and interact locally among themselves and react swiftly in time sensitive scenarios to the changing environmental conditions.

Paper [29] classified relocation algorithms, apart from their span of design overlap and similarity into virtual forcebased (radial [22], [33] or angular [26]), voronoi-based [27] and flip-based [21] movement algorithms. Regardless of the classification, each of the relocation algorithms is devised to reach performance goals (i.e. connectivity [26], lifetime [25], deployed node re-alignment [22], [29]-[32], small and large scale coverage holes recovery [21], [27], [29], virtual coverage hole displacement [34], etc.). However, whether these relocation algorithms have agreeable performance for other objectives than their primary design goals should be investigated in further details.

Although DSSA has a good performance with regard to amount of node movements and coverage [22] when it is applied to balance and align the node formations, it is shown in this paper that DSSA is not able to properly recover large scale coverage holes with the sufficient number of iterations even if all nodes participate in the recovery processes. In another scenario which DSSA only is applied to limited or select a set of nodes close (proximate)to the coverage hole (e.g. $\mathrm{CH}$ boundary nodes) [29], [35], [36] to limit node movements and power consumptions, DSSA ability of recovery worsen.

\section{Methods And Assumptions}

In our scenario, homogeneous sensor nodes based on model of the unit disk graph (UDG) [37] randomly deployed with uniform distribution in a rectangular area of $\left[x_{\min }, x_{\max }\right] \times\left[y_{\min }, y_{\max }\right]$. For sake of simplicity, nodes' transmission ranges $\left(R_{c}\right)$ and sensing ranges $\left(R_{s}\right)$ are equal. Nodes are bidirectionally connected if they are within each other's ranges. Locations of sensors may be known by GPS or any other localisation methods [38]. Similar to [29], [35], [36], sensor nodes are classified into into damaged nodes if they reside inside the damaged area (coverage hole); otherwise, they are considered as undamaged nodes. Those undamaged nodes proximate to the coverage hole which directly detect the damage event within their ranges are further defined as boundary nodes (Fig. 1). Coverage hole event is detected by boundary nodes as boundary nodes sense any significant changes within their ranges. (i.e. signal loss or disconnection as the result of the failure of their neighbours)

Similar to [29], [35], [36], coverage hole is modelled as a circle of radius $r_{\text {Hole }}$ with the centre at $x_{\text {Hole }}, y_{\text {Hole }}$ (Fig. 1). In this paper, Distributed Self-Spreading Algorithm (DSSA) [22], as one of force-based relocation algorithms with the promising performance in movement, uniformity and coverage, is applied to both boundary nodes and undamaged nodes in the network and their performances in term of coverage and the ability to repair damaged area is depicted in Figs. 2, 3. Similar to [30]-[32], nodes stop at the boundaries if their new locations obtained by relocation algorithm exceed the boundaries of the given area $\left(\left[x_{\min }, x_{\max }\right] \times\left[y_{\min }, y_{\max }\right]\right)$.

\section{RESUlTS}

The model is simulated in Matlab and $N=500$ nodes with transmission and sensing range of $R_{c}=R_{s}=15$ deployed in the rectangular 2-D area of $[-100,100] \times[-100,100] \mathrm{m}^{2}$. By considering boundary condition used in [30]-[32], DSSA applied to boundary nodes and undamaged nodes in the given area. Figs. 2, 3 show network deployment and coverage status before and after occurrence/recovery of coverage hole as DSSA algorithm performed on boundary and undamaged nodes in iteration $i=50$. It should be noted that the most recovery is resulted form first 10 iterations. As the number of iterations increases the DSSA performance does not improve accordingly. Therefore according to Figs. 2, 3, although DSSA is an efficient relocation algorithm for aligning unbalanced deployments or repairing of small coverage holes, it is a not proper choice for recovering the large scale coverage holes.

\section{CONCLUSION AND FUture WORK}

In this paper as one of force-based relocation algorithms, DSSA is chosen for recovery of large coverage hole. DSSA recovery capability and efficiency in term of coverage has been demonstrated as it is applied to both boundary and undamaged nodes. It is shown that DSSA is not proper choice for recovery of large scale coverage hole even if all network's nodes participate in the recovery process and/or the number of relocation iterations are increased sufficiently. As the possible future work, clear definition of small and large scale coverage holes can be presented. Recovery capability and efficiency of other conventional relocation algorithms in the case of small/large coverage hole can be investigated and their results can compared quantitatively in terms of different performance metrics.

\section{ACKNOWLEDGMENT}

This research was supported by the Australian Research Council (ARC) discovery research grant No. DP0879507. 


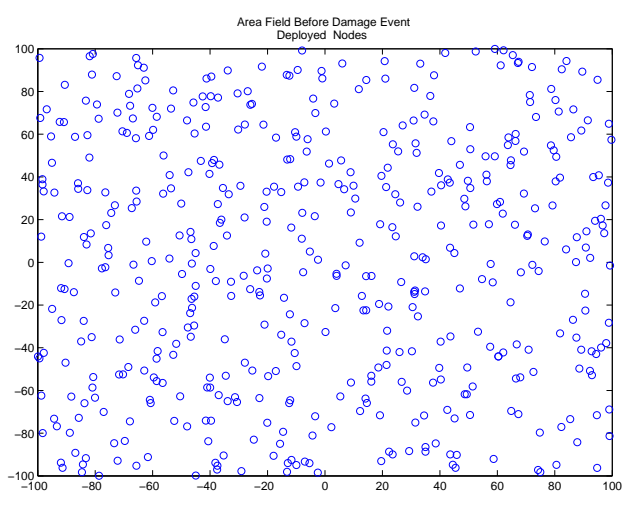

(a) Initial Network Deployment

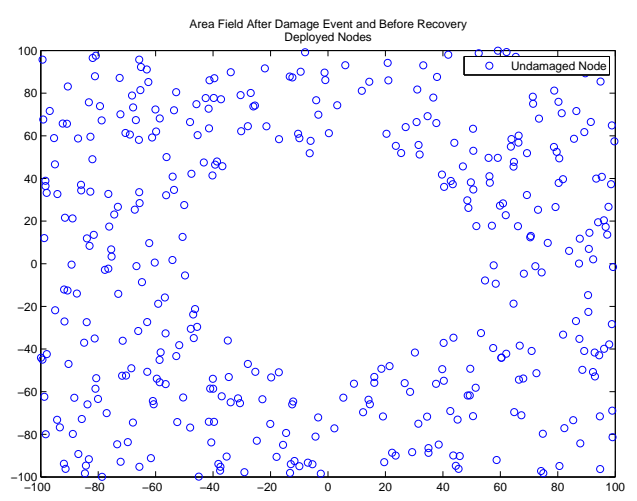

(b) Network Deployment After Coverage Hole/Before Recovery

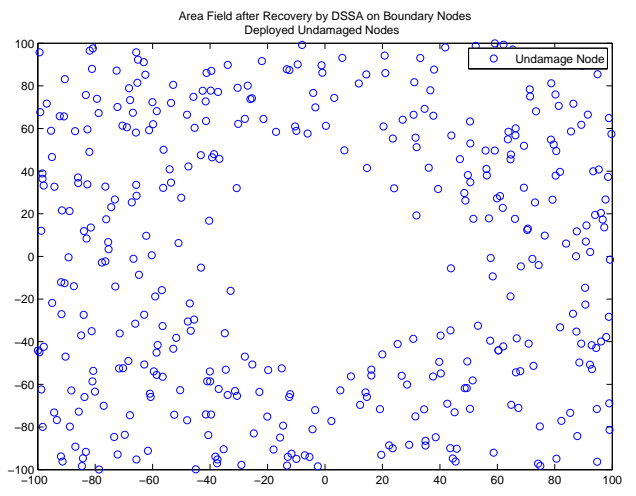

(c) Network Deployment After Recovery using Boundary Nodes

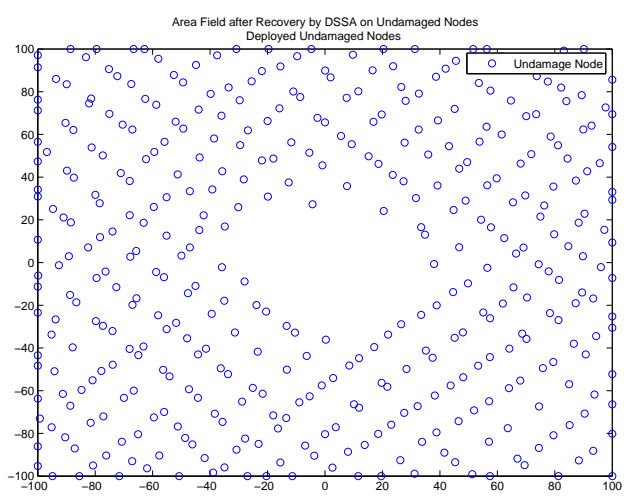

(d) Network Deployment After Recovery using Undamaged Nodes

Fig. 2: Network Deployment Stages in the Recovery, Performed by DSSA on Boundary/Undamaged Nodes

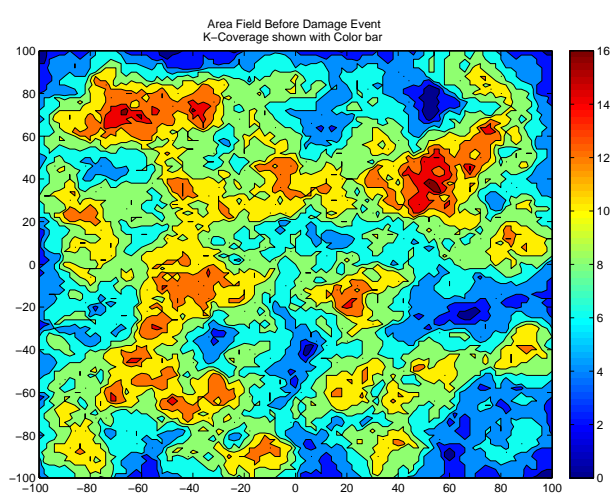

(a) Initial Network Coverage

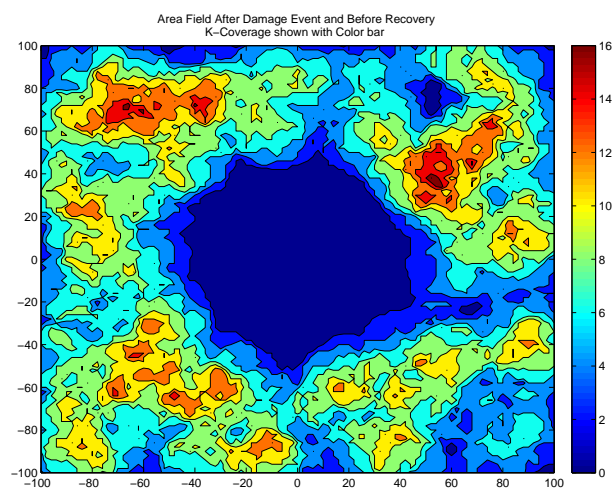

(b) Network Coverage After Coverage Hole/Before Recovery

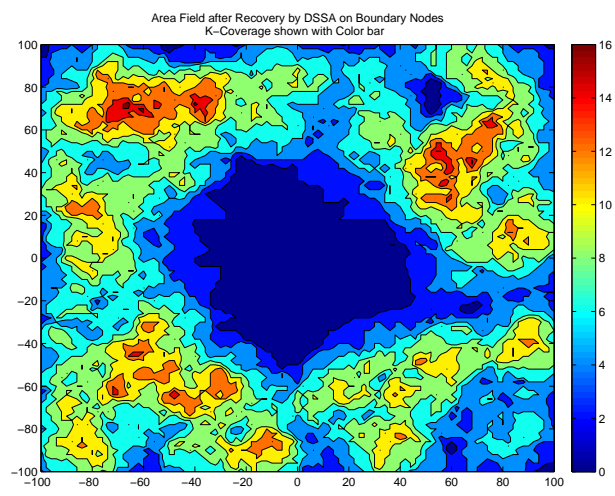

(c) Network Coverage After Recovery using Boundary Nodes

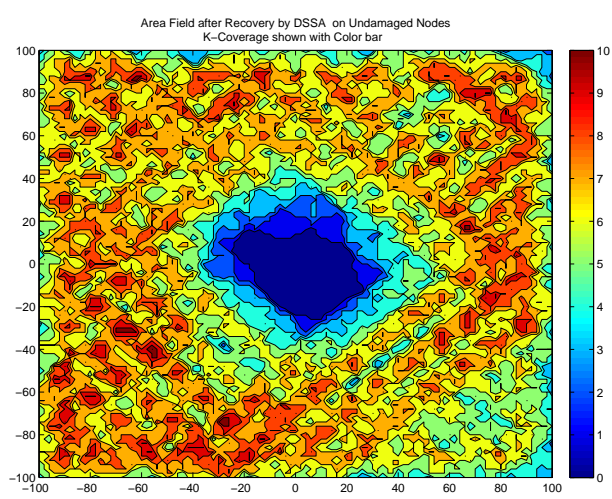

(d) Network Coverage After Recovery using Undamaged Nodes

Fig. 3: Network Coverage Stages in the Recovery, Performed by DSSA on Boundary/Undamaged Nodes 


\section{REFERENCES}

[1] I. Akyildiz, W. Su, Y. Sankarasubramaniam, and E. Cayirci, "Wireless sensor networks: a survey," Computer Networks, vol. 38, no. 4, pp. 393 $-422,2002$

[2] J. Yick, B. Mukherjee, and D. Ghosal, "Wireless sensor network survey," Computer Networks, vol. 52, no. 12, pp. 2292 - 2330, 2008.

[3] A. Khadivi and M. Hasler, "Fire detection and localization using wireless sensor networks," in Sensor Applications, Experimentation, and Logistics, ser. Lecture Notes of the Institute for Computer Sciences, Social Informatics and Telecommunications Engineering, N. Komninos, O. Akan, P. Bellavista, J. Cao, F. Dressler, D. Ferrari, M. Gerla, H. Kobayashi, S. Palazzo, S. Sahni, X. S. Shen, M. Stan, J. Xiaohua, A. Zomaya, and G. Coulson, Eds. Springer Berlin Heidelberg, 2010, vol. 29 , pp. 16-26.

[4] S. Bhima, A. Gogada, and R. Garimella, "A tsunami warning system employing level controlled gossiping in wireless sensor networks," in Proceedings of the 4th international conference on Distributed computing and internet technology, ser. ICDCIT 2007. Berlin, Heidelberg: Springer-Verlag, 2007, pp. 306-313.

[5] Y. Li, Z. Wang, and Y. Song, "Wireless sensor network design for wildfire monitoring," in The Sixth World Congress on Intelligent Control and Automation, WCICA 2006, vol. 1, 2006, pp. 109 -113.

[6] M. Suzuki, S. Saruwatari, N. Kurata, and H. Morikawa, "A highdensity earthquake monitoring system using wireless sensor networks," in Proceedings of the 5th international conference on Embedded networked sensor systems, ser. SenSys 2007. New York, NY, USA ACM, 2007, pp. 373-374.

[7] A. Mainwaring, D. Culler, J. Polastre, R. Szewczyk, and J. Anderson, "Wireless sensor networks for habitat monitoring," in Proceedings of the 1st ACM international workshop on Wireless sensor networks and applications, ser. WSNA 2002. New York, NY, USA: ACM, 2002, pp. 88-97.

[8] T. Arici and Y. Altunbasak, "Adaptive sensing for environment monitoring using wireless sensor networks," in Wireless Communications and Networking Conference, 2004. WCNC. 2004 IEEE, vol. 4, March 2004, pp. 2347-2352 Vol.4.

[9] G. Werner-Allen, K. Lorincz, M. Ruiz, O. Marcillo, J. Johnson, J. Lees, and M. Welsh, "Deploying a wireless sensor network on an active volcano," Internet Computing, IEEE, vol. 10, no. 2, pp. 18-25, March-April 2006.

[10] S. Kim, S. Pakzad, D. Culler, J. Demmel, G. Fenves, S. Glaser, and M. Turon, "Health monitoring of civil infrastructures using wireless sensor networks," in Proceedings of the 6th international conference on Information processing in sensor networks, ser. IPSN 2007. New York, NY, USA: ACM, 2007, pp. 254-263.

[11] M. Li and Y. Liu, "Underground structure monitoring with wireless sensor networks," in Proceedings of the 6th international conference on Information processing in sensor networks, ser. IPSN 2007. New York, NY, USA: ACM, 2007, pp. 69-78.

[12] K. Daniel, S. Rohde, and C. Wietfeld, "Leveraging public wireless communication infrastructures for uav-based sensor networks," in 2010 IEEE International Conference on Technologies for Homeland Security (HST), Nov. 2010, pp. 179-184.

[13] I. F. Akyildiz, D. Pompili, and T. Melodia, "Underwater acoustic sensor networks: research challenges," Ad Hoc Networks, vol. 3, no. 3, pp. 257-279, 2005.

[14] M. Jiang, Z. Guo, F. Hong, Y. Ma, and H. Luo, "Oceansense: A practical wireless sensor network on the surface of the sea," in IEEE International Conference on Pervasive Computing and Communications, 2009. PerCom 2009, March 2009, pp. 1-5.

[15] C. Xiao, Y. Peng, and M. Yu, "The deployment method and movement control strategy in mobile wireless sensor networks," in International Symposium on Computer Science and Computational Technology, ISCSCT 2008, vol. 2, Dec. 2008, pp. 520-523

[16] N. Ahmed, S. S. Kanhere, and S. Jha, "The holes problem in wireless sensor networks: a survey," SIGMOBILE Mob. Comput. Commun. Rev., vol. 9, no. 2, pp. 4-18, 2005

[17] J. Luo, D. Wang, and Q. Zhang, "Double mobility: coverage of the sea surface with mobile sensor networks," SIGMOBILE Mob. Comput. Commun. Rev., vol. 13, pp. 52-55, June 2009.

[18] B. Wang, H. B. Lim, and D. Ma, "A survey of movement strategies for improving network coverage in wireless sensor networks," Computer Communications, vol. 32, no. 1314, pp. 1427-1436, 2009.
[19] S. Toumpis, "Mother nature knows best: A survey of recent results on wireless networks based on analogies with physics," Computer Networks, vol. 52, no. 2, pp. 360 - 383, 2008

[20] B. Liu, P. Brass, O. Dousse, P. Nain, and D. Towsley, "Mobility improves coverage of sensor networks," in Proceedings of the 6th ACM international symposium on Mobile ad hoc networking and computing, ser. MobiHoc 2005. New York, NY, USA: ACM, 2005, pp. 300-308.

[21] S. Chellappan, X. Bai, B. Ma, D. Xuan, and C. Xu, "Mobility limited flip-based sensor networks deployment," IEEE Transactions on Parallel and Distributed Systems, vol. 18, no. 2, pp. 199-211, Feb. 2007.

[22] N. Heo and P. Varshney, "A distributed self spreading algorithm for mobile wireless sensor networks," in Wireless Communications and Networking, 2003. WCNC 2003. 2003 IEEE, vol. 3, March 2003, pp. $1597-1602$ vol.3

[23] M. Younis and K. Akkaya, "Strategies and techniques for node placement in wireless sensor networks: A survey," Ad Hoc Networks, vol. 6, no. 4, pp. $621-655,2008$.

[24] Y. Zou and K. Chakrabarty, "Sensor deployment and target localization in distributed sensor networks," ACM Trans. Embed. Comput. Syst., vol. 3, pp. 61-91, February 2004.

[25] Y. Yoo and D. Agrawal, "Mobile sensor relocation to prolong the lifetime of wireless sensor networks," in Vehicular Technology Conference, 2008. VTC Spring 2008. IEEE, may 2008, pp. 193 -197.

[26] A. Casteigts, J. Albert, S. Chaumette, A. Nayak, and I. Stojmenovic and, "Biconnecting a network of mobile robots using virtual angular forces," in Vehicular Technology Conference Fall (VTC 2010-Fall), 2010 IEEE 72nd, Sept. 2010, pp. $1-5$

[27] M. Argany, M. Mostafavi, and F. Karimipour, "Voronoi-based approaches for geosensor networks coverage determination and optimisation: A survey," in 2010 International Symposium on Voronoi Diagrams in Science and Engineering (ISVD), June 2010, pp. 115-123.

[28] W.-H. Liao, Y. Kao, and Y.-S. Li, "A sensor deployment approach using glowworm swarm optimization algorithm in wireless sensor networks," Expert Systems with Applications, vol. 38, no. 10, pp. 12180 - 12188 , 2011.

[29] A. Rafiei, M. Abolhasan, D. Franklin, and F. Safaei, "Wsns coverage hole partial recovery by nodes' constrained and autonomous movements using virtual alpha-chords," in ICWMC 2012, The Eighth International Conference on Wireless and Mobile Communications, 2012, pp. 74-80.

[30] Y. Maali, A. Rafiei, M. Abolhasan, D. R. Franklin, and F. Safaei, "A fuzzy logic node relocation model in wsns," in 6th International Conference on Signal Processing and Communication Systems 12 - 14 December 2012, Gold Coast, Australia, 2012.

[31] A. Rafiei, Y. Maali, M. Abolhasan, D. R. Franklin, F. Safaei, and S. Smith, "A tuned fuzzy logic relocation model in wsn using particle swarm optimization," in To be appeared in 2013 IEEE 78th Vehicular Technology Conference: VTC2013-Fall 2-5 September 2013, Las Vegas, USA, September 2013.

[32] A. Rafiei, Y. Maali, M. Abolhasan, D. R. Franklin, and S. Smith, "An iteratively tuned fuzzy logic movement model in wsn using particle swarm optimization," in Unpublished.

[33] Y. Zou and K. Chakrabarty, "Sensor deployment and target localization based on virtual forces," in INFOCOM 2003. Twenty-Second Annual Joint Conference of the IEEE Computer and Communications. IEEE Societies, vol. 2, March-3 April 2003, pp. 1293 - 1303 vol.2.

[34] C.-Y. Chang, W.-C. Chu, C.-Y. Lin, and C.-F. Cheng, "Energy-balanced hole-movement mechanism for temporal full-coverage in mobile wsns," in Proceedings of the 6th International Wireless Communications and Mobile Computing Conference, ser. IWCMC 2010. New York, NY, USA: ACM, 2010, pp. 89-93.

[35] A. Rafiei, "Boundary node selection algorithms by simple geometrical properties in wsns," in Fifth Asia Modelling Symposium (AMS), 2011, May 2011, pp. $221-226$.

[36] A. Rafiei, M. Abolhasan, D. Franklin, and F. Safaei, "Boundary node selection algorithms in wsns," in The 36th IEEE Conference on Local Computer Networks (LCN), 2011, October 2011, pp. 255-258.

[37] S. Schmid and R. Wattenhofer, "Algorithmic models for sensor networks," in 20th International Parallel and Distributed Processing Symposium, IPDPS 2006, April 2006.

[38] G. Mao, B. Fidan, and B. D. Anderson, "Wireless sensor network localization techniques," Computer Networks, vol. 51, no. 10, pp. 2529 - 2553, 2007. 